\title{
Photoprotection and skin self-examination in primary attention users: the impact of smartphone as a tool for education
}

\begin{abstract}
Introduction: "mobile health" consists in using electronic devices to support health.

Objectives: observe photoprotection, skin self-examination and compare the impact of multimedia presentation to Whatsapp messages on these habits in primary attention.

Methodology: experimental study, envolving 300 users. First moment: SEPI (Sun Exposure and Protection Index) and SSEAS (Self Skin-Examination Attitudes Scale) were applied and a presentation was performed. Second moment ( 8 weeks):150 users received photoprotection and self-examination messages. Third moment:300 re-answered questionnaires. Differences were analysed using $t$ test andlinear regression.
\end{abstract}

Results: $39.66 \%$ used sunscreen always/frequently and, in intervention, ascended to $47.91 \%(\mathrm{p}=0.0014)$ without change in control. Self-examination was a priority for $48.67 \%$ and increased to $73.91 \%$ in control $(\mathrm{p}=0.0179)$ and $69.8 \%(\mathrm{p}=0.0001)$ in intervention, without difference.

Conclusion: photoprotection remained low and self-examination became priority for twothirds (without difference between groups).

Keywords: mobile health, skin cancer, photoprotection
Volume 4 Issue 4 - 2020

\author{
Ricardo Tadeu Villa,' Ana Carolina Fortes \\ Braga Brederodes Villa,' Maria do Rosário \\ da Silva Ramos Costa, ' Ana Beatriz Costa \\ Brito, ${ }^{2}$ Brenna Carolina Sousa Braga, ${ }^{2}$ Eliana \\ Mika Yamaguchi, ${ }^{3}$ Magnus Falk ${ }^{4}$ \\ 'Professor UFMA, Federal University of Maranhão, Brazil \\ ${ }^{2}$ Student UFMA, Federal University of Maranhão, Brazil \\ ${ }^{3}$ Master UNIFESP, Federal University of São Paulo, Brazil \\ ${ }^{4}$ Professor LiU, University of Linköping, Sweden
}

Correspondence: Ricardo Tadeu Villa, Professor UFMA,
Federal University of Maranhão, Brazil, Email ricardovlladermato@hotmail.com

Received: August 08, 2020 | Published: August 31, 2020

\section{Introduction}

The use of mobile phones has grown rapidly. ${ }^{1}$ In many countries, the number of telephone lines exceeds the number of inhabitants and, in developing countries, the number of smartphones and the structure of telephony itself expands faster than other elements of infrastructure. The "mobile" nature of telephone, instant access, and direct communication allow the rapid transfer of health information, which supports medical and public health practices, and these are the defining characteristics of "mobile health" or "mHealth".

The popularization of mobile devices has been considered by many authors as the technological revolution with the greatest impact in recent years. ${ }^{3}$ In health, for example, there are applications for smartphones being used for behavioral change, that promote physical activity, ${ }^{4,5,6}$ facilitate the control of diseases such as diabetes ${ }^{7,8}$ and childhood obesity. ${ }^{9}$ With regard to Dermatology, there are applications that, through geolocation, can calculate the ultraviolet index of a certain area and the synthesis of vitamin D and dermatological atlases and applications that simulate skin diseases and provide education for students. ${ }^{10}$

Specifically, regarding the use of smartphones in sun protection education and skin self-examination, a study was conducted at the University of Colorado, ${ }^{11}$ and showed that reminders sent by smartphone resulted in greater adherence to photoprotection. This Colorado study represented a milestone in Dermatology, since there is a clear dissociation between what patients know about photoprotection and skin cancer and how effectively patients practice photoprotection and self-examination. In this regard, reminders sent by smartphone can be the decisive stimulus, transforming knowledge about photoprotection into actual photoprotection, increasing the social support for healthy behaviors and the responsibility of the users, transmitting emotional support ${ }^{12}$ and promoting the sense of will, choice and control. ${ }^{13}$ In this way, it is justified to know the habits of photoprotection and self-examination of primary care users and the impact of smartphone on health education.

The general objective of the present research is to observe the behavior of photoprotection and skin self-examination in primary care users and evaluate the impact of smartphone as an education tool. Additionally, specific objectives comprise: analyze whether there have been changes in photoprotection and self-examination after oral presentation supported by images; analyze whether there have been changes in photoprotection and self-examination after oral presentation supported by images and regular messaging; and, finally compare the results of the group in which there was only the oral presentation supported by images with the group that additionally received regular messages.

\section{Methodology}

\section{Type, period and sampling}

This was an experimental study aimed to evaluate the level of knowledge of users of a primary care unit about the importance of photoprotection and skin self-examination and the impact of smartphone as an educational tool.Once it was approved by Dutra Hospital Ethic Committee, its first moment was conducted during four weeks of July, 2018, monday to friday, and took place in a primary care unit, in São Luís. At this unit, the total number of new consultations is 300 weekly. 
Adults of both sexes, 18 years old or older, literate, smartphone owner with internet access, WhatsApp user and who consented to receive telephone messages were included and recruited, while waiting medical consultation, by verbal invitation. Systematically, 15 users were chosen each day, in the five days of weekly operation of the unit.

\section{Instruments}

Two questionnaires were the instruments used in this research: SEPI (Sun Exposure and Protection Index) and SSEAS (Skin SelfExamination Attitude Scale). Those are validated questionnaires and even the translations used is this research are validated by a board of specialists and have an alpha of Cronbach showing internal consistency.

SEPI questionnaire has two parts: SEPI 1, which maps exposure and protection habits, includes eight questions based on the fivedegree Likert scale ( 0 to 4 points), resulting in a total score ranging from zero to 32 points and the higher the exposure risk, the higher the score; and, SEPI 2, which maps the propensity to increase photoprotection, includes five questions ( 0 to 4 points), resulting in a total score ranging from zero to 20 , reflecting a greater propensity to enhance photoprotection. ${ }^{14}$

SSEAS, in its turn, includes a list of 10 questions, answered on a five-point Likert scale. The total SSEAS score ranges from zero to 40 , and zero indicates low and 40, high self-examination attitudes. ${ }^{15}$

Finally, demographic variables such as age, sex, education and phototype were recorded in a short direct questionnaire.

\section{Moments of the study}

\section{Initial moment: direct contact with the user}

Along 4 weeks, interviews were conducted and the data obtained (demographics, phone number and SEPI and SSEAS responses) were registered on tablets, in aonline form. Immediatelly after that, an oral, individual presentation, supported by images, was performed, addressing themes previously focused on SEPI and SSEAS.

\section{Intervention: WhatsApp messages}

Half of the subjects (150) were randomized to receive messages (short videos), addressing SEPI and SSEAS items, sent to the cell phone numbers collected at the initial moment. This stage lasted eight weeks and each subject received 16 messages, always on friday afternoons and saturday mornings.

\section{Follow-up: re-application of SEPI and SSEAS}

At the end of eight weeks, all 300 users received a link that directed them to the same on line questionnaires answered at the time of initial direct contact.

\section{Data and statistical processing}

For data analysis, Stata version 14 for Windows was used. Categorical variables were characterized in simple frequency tables, while continuous variables (age, SEPI 1, SEPI 2 and SSEAS scores) were described in terms of central tendency and variance.

Statistical differences were analyzed between group means using unpaired Student's t-test for continuous variables. Score trends were determined for the participants 'age,' 'sex', 'phototype' and 'education' by simple linear regression. In all analyzes, a significance level of $\mathrm{p}<0.05$ was used.

\section{Results}

\section{Characteristics of the studied population}

All 300 users answered the questionnaires and received information about skin cancer, photoprotection and self-examination, with no losses at the initial moment, for example, due to internet signal or other technical failures.

In the second moment, after eight weeks, there were losses. The intervention group had a $36 \%$ loss, since only 96 of the 150 users answered the questionnaires. The control group had a greater loss: only 46 answered the questionnaires, accounting for $69.33 \%$ of losses.

In the composition of the initial sample, there was a predominance of women $(63 \%)$, with phototypes $3(36.7 \%)$ and $4(30.5 \%)$, and mean age of 32 years and standard deviation of 13.97. Regarding education, individuals with complete high school (28\%) and incomplete college (32\%) predominated.

\section{Results of the questionnaires}

\section{SEPI 1}

Initial: pre-intervention assessment.

Prior to the intervention, when assessed for their UV exposure, respondents obtained a mean score of 11.47 with standard deviation 4.66 .

Control group: evaluation after eight weeks without messages.

Respondents who received only face-to-face guidance and were re-evaluated after eight weeks obtained a mean score of 9.63 with a standard deviation of 4.09 . This average was lower than the initial one with statistical significance $(\mathrm{p}<0.05)$.

Intervention group: evaluations after eight weeks of receiving messages.

Respondents who received weekly messages (in addition to instruction at the first moment of the study) and were re-evaluated after eight weeks, obtained a mean score of 8.93 with a standard deviation of 4.31. This average was lower than the initial one with statistical significance $(p<0.05)$. However, this mean and that of the control group showed no statistical difference between them (Figure 1).

\section{SEPI 2}

Initial: pre-intervention assessment.

Prior to the intervention, when evaluated for propensity to increase sun protection, users obtained a mean score of 10.81 with standard deviation 4.04 .

Control: evaluation after eight weeks without messages.

Respondents who received only face-to-face guidance and were re-evaluated after eight weeks obtained a mean score of 12.6 with a standard deviation of 3.9. This average was higher than the initial one with statistical significance $(\mathrm{p}<0.05)$.

Intervention: evaluations after eight weeks of receiving messages.

Respondents who received weekly messages for eight weeks (in addition to instruction at the first moment of the study) and were re-evaluated after this period, obtained a mean score of 12.3 with a standard deviation of 3.55. This average was higher than the initial one with statistical significance $(\mathrm{p}<0.05)$. However, this average and 
that of the control group showed no significant difference between them (Figure 2).

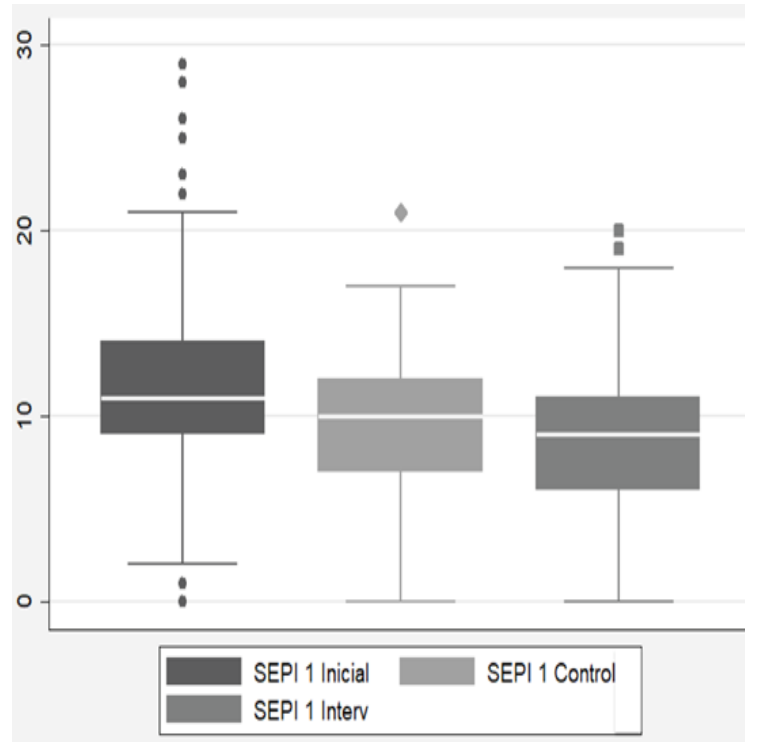

Figure I Box plot for visualization of SEPI grade I averages.

Note: Reduced number of outliers and reduced score after eight weeks in both receiving and non-receiving groups.

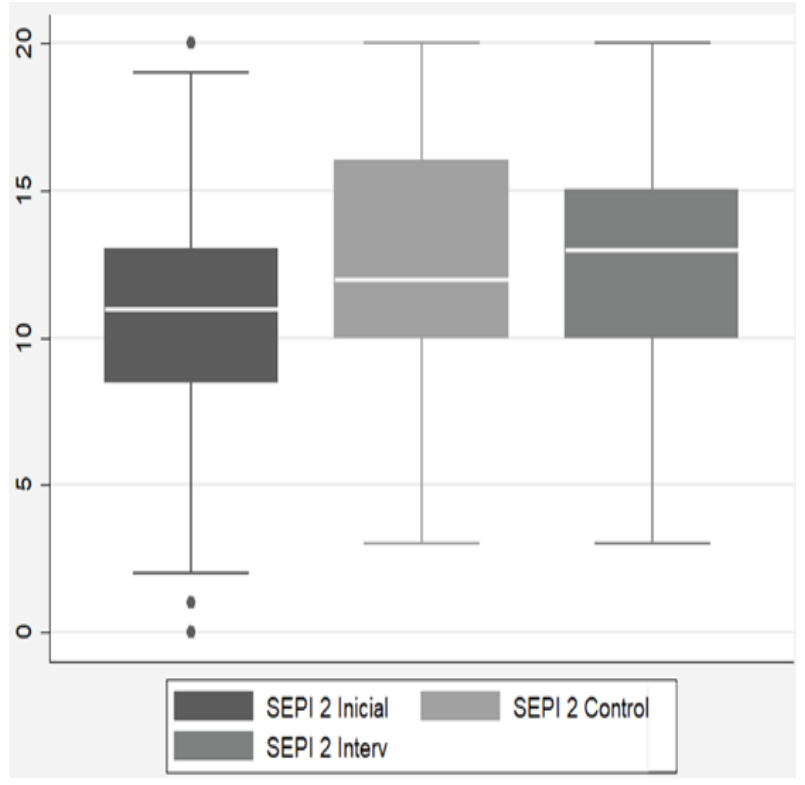

Figure 2 Box plot for visualization of SEPI 2 grade averages.

Note: Reduced number of outliers and increased score after eight weeks in both receiving and non-receiving groups.

\section{SSEAS}

Initial: pre-intervention assessment.

Prior to the intervention, when assessed for positive selfexamination attitude, respondents obtained a mean score 26.34 with standard deviation. . $^{6,7}, 9$

Control: evaluation after eight weeks without messages.

Respondents who received only face-to-face guidance and were re-evaluated after eight weeks had a mean score of 29.76 with a standard deviation of 5.03. This average was higher than the initial one with statistical significance $(\mathrm{p}<0.05)$.

Intervention: Evaluations after eight weeks of receiving messages.

Respondents who received weekly messages for eight weeks (in addition to instruction at the first moment of the study) and were reevaluated after this period, obtained a mean score of 29.31 with a standard deviation of 4.42. This average was higher than the initial one with statistical significance $(\mathrm{p}<0.05)$. However, this average and that of the control group showed no significant difference between them (Figure 3).

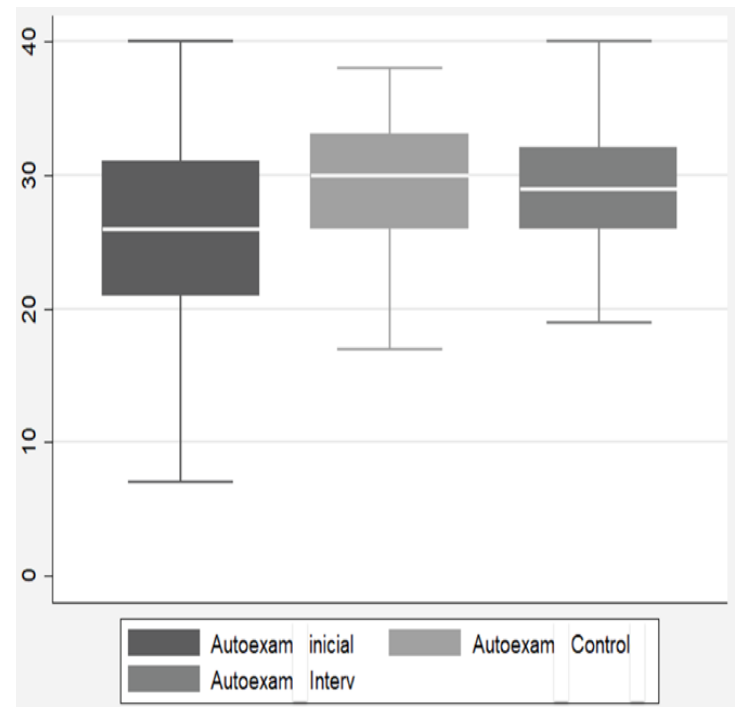

Figure 3 Box plot for SSEAS grade point visualization.

Note: No outliers and score increase after eight weeks in both receiving and nonreceiving groups.

\section{Results of some isolated items from questionnaires}

In the following section, individual questions are identified by the abbreviation of the questionnaire name, underline ( $\_$), and question number. Thus, for example, SSEAS question number 3 is identified by "SSEAS_3".

Therefore, SEPI 1_5 question revealed that, regarding photoprotection, $39.66 \%$ of users mentioned using sunscreen always or often at the beginning. In the intervention group, this value rose to $47.91 \%(p=0.0014)$ and, in the control group, there was no change after eight weeks. The questions SEPI 1_6 and SEPI 1_7, respectively, showed that, initially, the use of long clothes was part of the routine of $13.67 \%$ and hats, of $19 \%$ of users. At the end of eight weeks, long clothes use increased to $22.88 \%$ in the intervention group $(\mathrm{p}=0.0000)$, but did not increased in the control group and hat use increased both in control $(36.96 \%$ with $\mathrm{p}=0.0044)$ and intervention group $(32.3 \%$ with $\mathrm{p}=0.0000$ ), but with no difference between these two groups. Initially, the habit of staying in covered areas to avoid the sun (SEPI1_8) was always or often adopted by $81 \%$ of users and remained stable in the control and intervention groups.

Concerning SSEAS, the SSEAS 1question revealed that a high percentage of users $(92.33 \%)$ knew the importance of skin self-examination (SSEAS_1) at the initial time of the survey and this number remained stable after eight weeks in both groups. However, although this significant contingent of users knew the selfexamination, SSEAS_3 question revealed that initially less than half of them $(48.67 \%)$ considered their skin to be a priority, and by the end 
of eight weeks this percentage increased to $91 \%$ in control $(\mathrm{p}=0.0179)$ and $69.8 \%(\mathrm{p}=0.0001)$ in intervention group, with no difference between groups.

The ability to identify a suspected skin lesion was assessed in SSEAS 4 question, and, at the beginning, $57 \%$ of users declared themselves capable and this number increased to $72.92 \%$ in intervention group ( $\mathrm{p}=0.0087$ ), but not in control group. For its turn, SSEAS_ 5 question revealed that $78 \%$ of users would go to the doctor if they identified a suspicious lesion at the beginning of the study and this number reached $95.65 \%(\mathrm{p}=0.0010)$ in control group and $91.66 \%(\mathrm{p}=0.0101)$ in intervention group with no statistical difference between the groups.

Finally, the SSEAS 6 question showed that the user trusts the physician's ability, with positive assessments by more than $70 \%$ of respondents at all times in the survey.

\section{Discussion}

Internet and its related devices are redefining the educational process by providing dynamic and efficient channels for the interaction and transmission of information between individuals and institutions. The present study addressed this phenomenon in Health area, and showed that digital sharing of knowledge with the user brought positive changes in their photoprotection and skin self-examation behaviors. In addition, this is the first study in Brazil to use WhatsApp to disseminate scientific information in Dermatology.

Regarding the demographic data of the sample, only education differs significantly from what was found in literature. In a recent article, it was observed that primary health users completed high school $(29.9 \%)$ or elementary $(53.7 \%)$ level. ${ }^{16}$ In the present study, however, there was a higher number of users with incomplete $(32 \%)$ and complete $(24.33 \%)$ college, making up $56.33 \%$ of the initial sample. Nonetheless, schooling was not statistically related to SEPI 1, SEPI 2 and SSEAS scores, either at baseline or in the control and intervention groups. Thus, it was confirmed that $m$ Health can impact the most different population groups, regardless of their level of education.

However, the positive impact independent of education does not mean that mHealth should be thought outside the context of education as a whole. In fact, among the social determinants of health, education is fundamental, representing a powerful way to break the cycle of poverty and promote equity in health and suggests that public policies should be made keeping in mind that investment in health should be accompanied by investments in education for more robust results. ${ }^{17}$

Concerning SEPI 1 score, it decreased in both control and intervention groups, indicating reduced risk of UV exposure, at eight weeks. On the other hand, the engagement showed great differences and $69.33 \%$ of the control group users stopped responding to SEPI 1 at the end of the study. In the intervention group, however, the losses were much lower (36\%). This behavioral pattern suggests that a minimum time of contact or a minimum repetition of contact is necessary to sensitize a certain contingent of users. Or put it another way: that one moment of applying the questionnaires and the explanation supported by images may be to short for establishing a good level of engagement.

On this issue of minimal interaction in Medicine, a study revealed that interactions between doctors and patients lasting at least 15 minutes translated into a better perception of patient care and even a better prognosis in chronic diseases such as diabetes. ${ }^{18}$ This event was also observed here, in the form of greater engagement among those users whose contact with the researcher, via Whatsapp, was sustained for eight weeks.

In addition, regarding this amount of interaction with the user, the literature does not clarify the ideal duration of the intervention or the most appropriate number of messages. However, in a recent Brazilian study, approaches with one to three messages per week and over a period of 8 to 12 weeks were more frequent. ${ }^{19}$ In this regard, it is worth noting that interventions with higher frequency of message sending or for longer periods did not bring more engagement or more expressive results. ${ }^{20,21}$

The second questionnaire applied was the SEPI 2 and the rise of its score at the final moment of both groups indicates that users can further increase photoprotection in the future. By the way, this predisposition to continue changing the photoprotection (which has already increased according to SEPI 1) can be explained by the transtheoretical model. According to this model, if the initial resistance to some change had been overcome, users would have overcome an "emotional barrier" and become more open for new changes to occur. ${ }^{22}$

Still regarding SEPI 2, there was the same pattern of engagement losses (higher in the control group). The fact that deserves to be highlighted is that, although the losses differed between the groups, the final SEPI 2 scores did not present statistical difference between the groups either.

Regarding age, there was no more prudent behavior regarding sun exposure (SEPI 1) or greater propensity to increase photoprotection (SEPI 2) in older users. This fact contradicts what is widely observed in literature: "over time, individuals in the process of aging, incorporate self-knowledge and attention to one's own health into their identity." ${ }^{23}$ Similarly, it was not observed that dark skinned individuals were more exposed to the sun (SEPI 1) or less prone to photoprotection (SEPI 2). This result was also unexpected, based on the fact that people with darker skin tend to protect themselves less from the sun, as they are naturally less subject to the development of skin cancers. ${ }^{24}$

It is also worth mentioning that in all initial scores, regarding gender, women scored better than men with statistical significance. This is not surprising, as women are usually more attentive to their own health. ${ }^{25}$ However, by the end of the study, again, the overarching and inclusive aspect of mHealth became evident: men and women were equally sensitized by the intervention and, after eight weeks, there was no longer a statistically significant difference in gender scores.

For its turn, SSEAS index varied positively in control and intervention groups, indicating more positive attitudes toward selfexamination at the end of eight weeks in both groups. However, as observed for SEPI, there was a large loss of engagement in control group for SSEAS, which did not imply statistical difference between the scores of the control and intervention groups. In addition, similarly to what was observed for SEPI, there was no correlation between SSEAS and age, education and phototype of users.

Keeping in mind one of the objectives of the present study (to observe the photoprotection situation), SEPI 1 revealed that $39.66 \%$ of users mentioned that they used sunscreen always or often at the beginning (SEPI 1_5). In the intervention group, this value rose to $47.91 \%$. This is a positive result for the intervention, with statistical significance and is very encouraging, since, according to research 
released by SBD in 2017, only $35 \%$ of Brazilians applied sunscreen frequently. ${ }^{26}$ For this SEPI $1 \_5$ question, there was no change in control group after eight weeks and a single encounter was not enough to change sunscreen habit.

Concerning the use of clothing (SEPI 1_6) and hats (SEPI 1_7),it was less frequent than the use of photoprotector, at the beginning, but staying in covered areas to avoid the sun (SEPI 1 8) was a habit always or often adopted by the majority of the users. At the end of eight weeks, the shadow-seeking habit remained stable in the control and intervention groups and the use of long clothes, after eight weeks, was a protection strategy for only one in five users and wearing hats was a habit for one in three users (low level of adherence to both). In this regard, as observed in Australia, it can be theorized that, also in São Luís, the high average temperatures throughout the year make wearing long clothes and hats uncomfortable and this photoprotection strategy will become a viable option only when lighter, ventilated and chemically treated to protect fabrics were affordable. ${ }^{27}$ With another objective in mind, it is important to mention that SSEAS_1 revealed that almost all users knew the importance of skin self-examination at the time of the initial survey and that remained stable after eight weeks in both groups. This data, when compared with the photoprotection habits of the initial sample (low use of sunscreen, long clothes and hats) is in accordance with that already mentioned in this study: there is a mismatch between what people know about skin cancer and selfexamination and their effective photoprotection habits.

Finally, item SSEAS_6 revealed that the user trusted in physician's ability, with positive evaluations by most respondents at all times of the research. It is also noteworthy that there was an increase in the number of individuals who stated that they would seek medical attention if they identified something suspicious on their skin in both control and intervention group (SSEAS_5). These results are in agreement with the CFM survey published in 2016: "In Brazil, doctors are the professional that the population trusts the most, and this is the perception of $26 \%$ of Brazilians, who secondly place teachers (24\% of mentions) and firefighters (15\%)." ${ }^{28}$

Although initially a significant number of users knew selfexamination, less than half of them considered it a priority to examine their own skin, but by the end of eight weeks this percentage increased significantly in both groups. In other words, there was a reinforcement for the self-examination initiative, fulfilling the educational objective of the study.

Finally, losses are very common in $\mathrm{m}$ Health studies and should not discourage this kind of intervention, taking into account benefits already mentioned. ${ }^{29}$

\section{Conclusion}

At the beginning of the study, it was observed that less than half of the users adhered to regular photoprotection measures and, although this habit became more frequent, after eight weeks, it remained below ideal. In fact, the only photoprotection strategy mostly adopted by patients at all times in the study was to avoid the sun by seeking shade.

Regarding skin self-examination, at all times of the study, almost all users recognized its importance, although in the initial moment, its realization was not a priority for most. By the time of eight weeks, self-examination had become a priority for two-thirds of users.

It is noteworthy that it was not possible to state that the use of messages via WhatsApp associated with oral presentation to users was more effective than oral presentation alone, as the scores of the SEPI 1, SEPI 2 and SSEAS questionnaires showed an improvement, with statistical significance in both control and intervention groups, with no difference between these groups.

Given these results, as smartphones, networks and platforms become more powerful, they will benefit more and more people around the world and, as mentioned by Kratzke: "health education and health promotion specialists need to understand how to incorporate smartphones and motivational apps such as patient reminders, patient education, behavior modification, patient compliance, or decision support. ${ }^{30}$

\section{Acknowledgments}

None.

\section{Conflicts of interest}

The author declares that there is no conflicts of interest.

\section{Funding}

None.

\section{References}

1. Carter A, Liddle J, Hall W, et al. Mobile Phones in Research and Treatment: Ethical Guidelines and Future Directions. JMIR Mhealth Uhealth. 2015;3(4):95.

2. Donner J. Research Approaches to Mobile Use in the Developing World: A Review of the Literature. The Information Society. 2008;24 (3):140 159.

3. Levy P. Cibercultura. São Paulo. Ed. 34. 1999. Disponível em.2018.

4. Kirwan M, Duncan MJ, Vandelanotte C. et al. Design, develop-ment, and formative evaluation of a smart phone application for recording and monitoring physical activity levels: The 10,000 steps "iStepLog'. Health Educ Behav. 2013;40 (2):140-151.

5. Rabin CE, Bock B. Desired features of smartphone applications promoting physical activity. Telemed J E Health. 2011;17(10): 801-803.

6. Zwerver J, Weir A, Tol JL. What's app? Sports medicine physicians should not talk double Dutch. Br J Sports Med. 2012;46 (12):833-834.

7. Fukuoka Y, Kamitani E, Bonnet K, et al. Real-time social support through a mobile virtual community to improve healthy behavior in overweight and sedentary adults: a focus group analysis. J Med Internet Res. 2011;13 (3):49-52.

8. Waki K, Fujita H, Uchimura Y, et al. Dial Betics: Smartphone-Based Self-Management for Type 2 Diabetes Patients. J Diabetes Sci Technol. 2012;6(4): 983-985.

9. Fassnacht DB, Ali K, Silva C, et al .Use of text messaging services to promote health behaviors in children. J Nutr Educ Behav. 2015; 47(1):7580 .

10. Brewer AE, Sampson DH, Sampson JA. Mobile Applications in Dermatology. JAMA Dermatology. 2013;149 (11):1300-1304.

11. Buller DB, Berwick M, Lantz K, et al. Smartphone Mobile Application Delivering Personalized, Real-Time Sun Protection AdviceA Randomized Clinical Trial. JAMA Dermatol. 2015;151(5):497-504.

12. Fjeldsoe B, Miller Y, Marshall A. Text messaging interventions for chronic disease management and health promotion. In: Noar S, Harrington N, eds. E-Health Applications: Promising Strategies for Behavior Change. New York, NY: Routledge. 2012:167-186. 
13. Westmaas JL, Bontemps-Jones J, Bauer JE. Social support in smoking cessation: reconciling theory and evidence. Nicotine Tob Res. 2010;12 (7):695-707

14. Detert H, Hedlund S, Anderson CD, et al. Validation of sun exposure and protection index (SEPI) for estimation of sun habits. Cancer Epidemiology. 2015;39:986-993.

15. Janda M, Youl PH, Lowe JB, et al. Attitudes and intentions in relation to skin checks for early signs of skin cancer. Prev Med. 2004;39(1):11-18.

16. Guibu IA, Moraes JC, Guerra AA, et al. Características principais dos usuários dos serviços de atençãoprimária à saúde no Brasil. Rev Saude Publica. 2017;51 Supl 2:17s.

17. Hahn RA, Truman BI. Education Improves Public Health and Promotes Health Equity. Int J Health Serv. 2015;45(4):657-678.

18. Dugdale DC, Epstein R, Pantilat SZ. Time and the Patient-Physician Relationship. J Gen Intern Med. 1999;14(Suppl 1):S34-S40.

19. Marcolino MS, Oliveira JAQ, D'agostino, M. et al. The Impact of mHealth Interventions: Systematic Review of Systematic Reviews. JMIR Mhealth Uhealth. 2018;6 (1):1-34.

20. Wargny M, Kleinebreil L, Diop SN. SMS-based intervention in type 2 diabetes: clinical trial in Senegal. BMJ Innov. 2018;4:142-146.

21. Drake AL, Unger JA, Ronen K, et al. Evaluation of mHealth strategies to optimize adherence and efficacy of Option $\mathrm{B}+$ prevention of motherto-child HIV transmission: Rationale, design and methods of a 3-armed randomized controlled trial. Contemporary Clinical Trials. 2017;57: 4450
22. Prochaska J, Velicier W, Redding, C. et al. Stage--based expert systems to guide a population of primary care patients to quit smoking, eat healthier, prevent skin cancer, and receive regular mammograms. Prev Med. 2005;41(2): 406-416.

23. Diehl M, Wahl HW, Barrett AE, et al. Awareness of Aging: Theoretical Considerations on an Emerging Concept. Dev Rev. 2014; 34 (2):93-113.

24. Gupta AK, Bharadwaj M. Skin Cancer Concerns in People of Color: Risk Factors and Prevention. Asian Pac J Cancer Prev. 2016;17(12): 52575264.

25. Stefan EK. Gender differences in health information behaviour: a finnish population-based survey. Health Promotion International. 2015;(30)3:736-745.

26. SBD. Fototipos de Pele. Disponível em. 2018.

27. Nikles J, Harrison SL. An Observational Study of Sun-Protective Behaviour at an Outdoor Spectator Sporting Event in a Region of High Sun Exposure. J Carcinog Mutagen. S4: 003. 2014.

28. CFM: levantamento mostra o médico como profissional com maior credibilidade e confiança junto aos brasileiros. Disponível 09. 2018.

29. Dugas M, Gao G, Agarwall R. Unpacking mHealth interventions: A systematic review of behavior change techniques used in randomized controlled trials assessing mHealth effectiveness. Digit Health. 2020;(6):1-16.

30. Kratzke C, Cox C. Smartphone Technology and Apps: Rapidly Changing Health Promotion. International Electronic Journal of Health Education. 2012;15:72-82 